\title{
Culture scientifique et territoires
}

\section{Samuel Cordier}

URL : http://journals.openedition.org/ocim/1299

DOI : 10.4000/ocim.1299

ISSN : 2108-646X

\section{Éditeur}

OCIM

Édition imprimée

Date de publication : 29 novembre 2013

ISSN : 0994-1908

\section{Référence électronique}

Samuel Cordier, "Culture scientifique et territoires », La Lettre de l'OCIM [En ligne], 150 | 2013, mis en ligne le 29 novembre 2015, consulté le 02 mai 2019. URL : http://journals.openedition.org/ocim/1299 ; DOI : 10.4000/ocim.1299

Ce document a été généré automatiquement le 2 mai 2019.

Tous droits réservés 


\section{Culture scientifique et territoires}

\section{Samuel Cordier}

L'auteur tient à remercier Jean-Yves Bernaud (conseiller scientifique au Pavillon des sciences) et Rémy Morel (directeur du CCSTI Lacq Odyssée) pour leurs relectures attentives.

1 Comment analyser l'évolution actuelle de la place de la culture scientifique technique et industrielle dans les territoires? Depuis 2011, l'émergence de projets soutenus par le Commissariat Général aux Investissements d'Avenir, la mise en œuvre de l'acte III de la décentralisation, prévoyant notamment d'accentuer le rôle des Régions, la mise en place 
d'une nouvelle gouvernance ${ }^{1}$ ou encore de nouveaux rythmes scolaires sont autant de facteurs qui dessinent un nouveau paysage de la culture scientifique et technique.

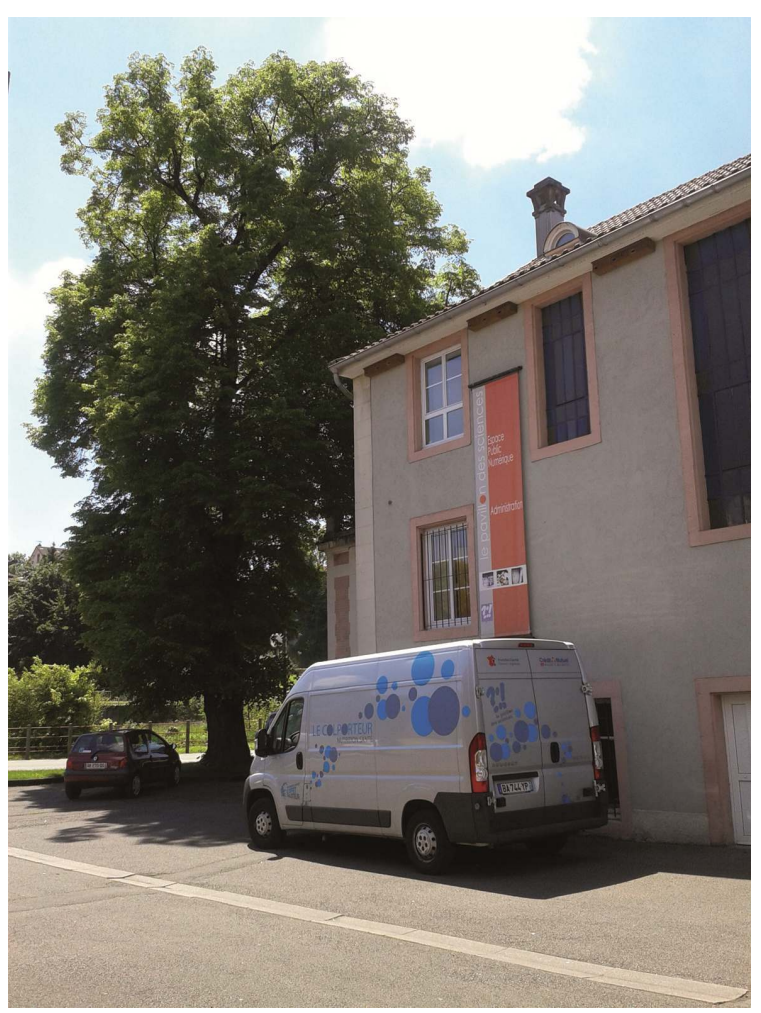

Le Colporteur des sciences est l'outil dont s'est doté le Pavillon des sciences pour remplir une mission régionale d'alphabétisation scientifique et intervenir plus particulièrement en milieu rural.

(c) PAVILLON DES SCIENCES

2 Bien avant que l'idée d'une culture scientifique technique et industrielle soit formulée, l'exemple des relations savantes au XVIII ${ }^{\mathrm{e}}$ siècle montre que des dispositifs efficaces de correspondances et d'échanges ont été développés dans l'Europe savante pour mailler le territoire. L'histoire récente, résultant autant d'actions militantes que de politiques publiques, prouve que les acteurs de la culture scientifique n'ont cessé d'innover pour conquérir de nouveaux territoires. Des pionniers de la "physique dans la rue », au développement actuel des sciences participatives, en passant par la création des Centres de Culture Scientifique Technique et Industrielle (CCSTI), ou le développement d'actions " hors les murs", des outils sans cesse renouvelés ont permis de toucher de nouveaux publics et territoires.

En 1984, Jean-Marc Levy-Leblond proposait dans le magazine La Recherche une définition de la culture scientifique et technique dans laquelle les rapports entre culture scientifique et territoires apparaissaient déjà clairement énoncés :

«Les objectifs de la culture scientifique et technique sont de mettre en culture les sciences et les techniques, d'encourager leur délocalisation, de faire éclater des territoires, de sourdre dans tous les champs d'activités et d'investissements sociaux: politique, économique, de la re-

cherche, de la formation, social, éthique..., de renouveler les approches de la société et du monde. Pour atteindre ces buts, obligation est requise d'utiliser tous les appuis, les forces vives et sensibles : l'école, la rue, l'usine, le laboratoire, le bureau, les équipements socioculturels et culturels, les clubs, les associations de jeunes... $»^{2}$. 
6 Les pratiques induites par des initiatives récentes à destination des publics «éloignés ", géographiquement comme sociologiquement, s'inscrivent dans la continuité des actions de culture scientifique conduites depuis plusieurs décennies. Il paraît également que si la place de la culture scientifique technique et industrielle dans les territoires peut être analysée à travers ses acteurs, ses réseaux ou ses outils, elle est envisagée aujourd'hui à partir des publics dans leurs environnements (géographique, économique, sociologique). Identifiées dans un projet de territoire, les actions développées contribuent de manière efficace à faire évoluer la relation des citoyens aux sciences.

\section{Aller à la rencontre du public}

7 Les années 1970 ouvrent une période au cours de laquelle émergent des initiatives visant à rapprocher les publics des centres de production du savoir. Ces mutations résultent à la fois d'une interrogation des chercheurs sur leur place dans la société et sur des changements économiques et politiques.

En effet depuis les années 1960, un nouveau réseau d'acteurs se structure sur l'ensemble du territoire. Une loi de juillet 1960 est à l'origine des Parcs nationaux et à partir de 1971, se développe le réseau des écomusées, « un miroir où cette population se regarde, pour s'y reconnaître, où elle recherche l'explication du territoire auquel elle est attachée, jointe à celle des populations qui l'ont précédée, dans la discontinuité ou la continuité des générations $»^{3}$.

9 Aux côtés des musées, des musées de sciences traditionnels, les acteurs de la médiation scientifique se multiplient et leurs actions à destination de nouveaux publics se diversifient. Alors que l'idée d'une culture scientifique technique et industrielle se concrétise, des associations investissent autant les zones urbaines intermédiaires que le milieu rural, les laboratoires des universités ouvrent leurs portes, les chercheurs vont à la rencontre des publics.

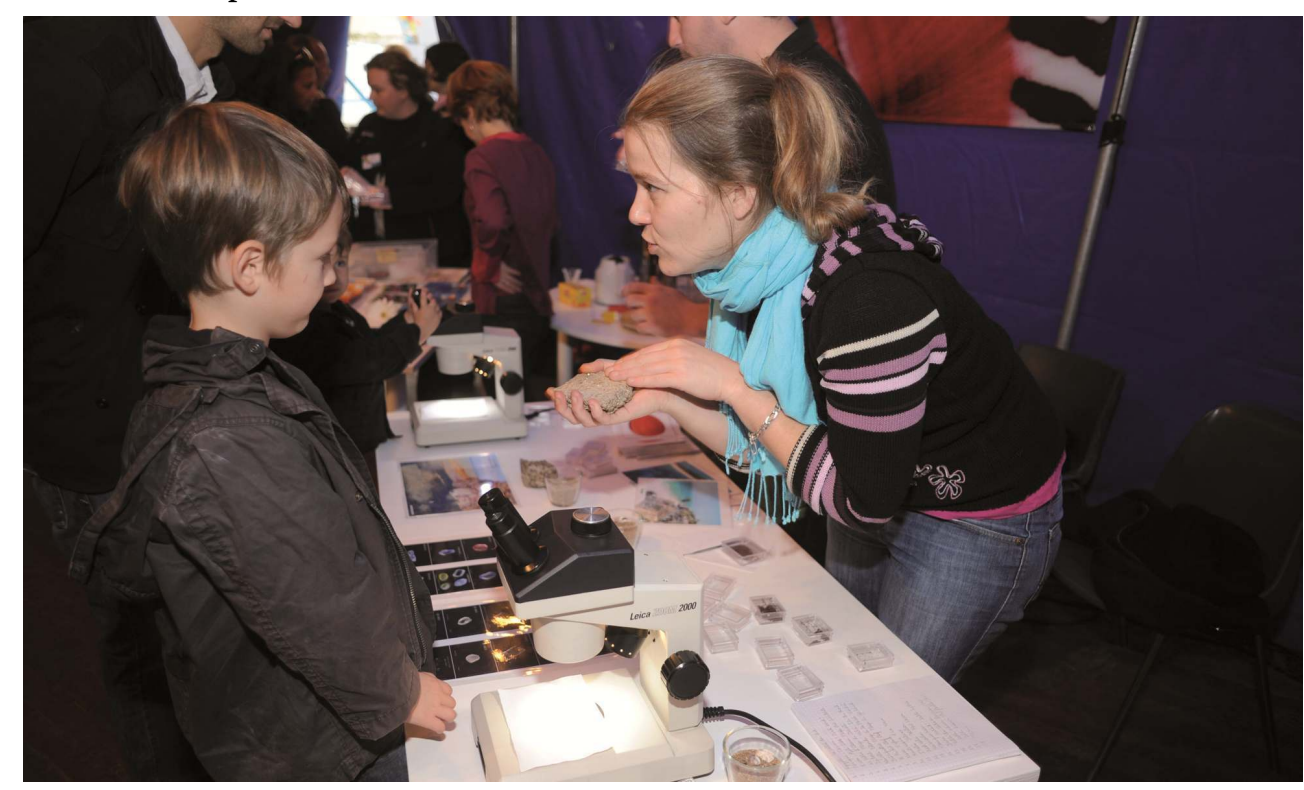

Animations proposées par le muséum d'Histoire naturelle de Nantes dans le cadre de la Fête de la Science.

(c) MUSÉUM DE NANTES 
actions (la création de l'Association nationale des clubs scientifiques (ANCS) ou de clubs d'amateurs autour des muséums) à destination des publics "hors les murs" existaient dans les années 1960, Étienne Guyon et Bernard Maitte montrent comment la réflexion de quelques chercheurs sur la place de leur domaine de recherche dans la société a favorisé l'émergence de nouvelles pratiques.

En 1973, à l'occasion d'un congrès international de physique des particules organisé à Aixen-Provence, le physicien Michel Crozon (1932-2008) et un collègue suisse, décident de présenter dans la rue des expériences de physiques générale et nucléaire. "Cette démarche reposait sur la juste prise de conscience par les chercheurs qu'il fallait informer (et écouter !) le public, en particulier celui qui ne va pas dans les musées, et sur le besoin de se justifier sur la légitimité d'une recherche scientifique non finalisée (les manifestations impliquaient presque exclusivement des chercheurs de sciences de base) » ${ }^{4}$. L'année suivante, en 1974, est fondée le Groupe de liaison pour une action culturelle scientifique (GLACS), une structure destinée à l'accompagnement d'actions culturelles en sciences.

Dans la suite de cette réflexion, les universités et les établissements publics de recherche vont développer des actions pour aller à la rencontre des publics dans les régions. À la fin des années 1970, des «Boutiques des sciences » ouvrent leurs portes dans les universités de Grenoble, Lyon, Paris, Strasbourg, puis au début des années 1980, à Lille, Marseille et Orsay. «Lieux de médiation, ces structures recevaient des demandes concrètes de la société civile (associations, entreprises), les formulaient en langage scientifique, trouvaient les interlocuteurs compétents pour y répondre ou pour mener les recherches nécessaires $»^{5}$. Les établissements publics de recherche développent également des actions, comme l'Inserm, avec ses «Clubs Inserm », à destination des lycéens.

\section{Décentralisation}

La création de lieux dédiés à la culture scientifique dans les régions est étroitement liée à l'entrée de la science dans les espaces culturels. Pour Andrée Bergeron, le développement de ce réseau résulte de la « la rencontre, principalement autour de la décennie 1970, entre une critique interne à la science et l'émergence en France d'un courant Science, technologie, société (STS) [...]» et à l'émergence des maisons de la culture; « conformément au projet d'André Malraux, les maisons de la culture s'ouvrent sur des projets culturels polyvalents qui, le plus souvent, prennent en compte la thématique scientifique $»^{6}$.

ier Centre de Culture Scientifique et Technique (CCST) ouvre ses portes en 1979 Grenoble dans les locaux de la Casemate, mis à disposition par la ville. Comme le rappellent Étienne Guyon et Bernard Maitte : « en 1966, à l'occasion de la préfiguration d'une Maison de la Culture à Grenoble, un groupe de scientifiques défend l'idée d'y intégrer une animation scientifique, au même titre que les autres activités, musicales, théâtrale, cinématographiques... son but est de faire admettre que les sciences sont un élément constitutif de la culture contemporaine ». C'est ainsi, qu'un secteur "sciences » sera mis en place dès l'ouverture de cette maison de la culture.

Le début des années 1980 marque un nouveau départ. "C'est l'arrivée de la gauche au pouvoir en 1981 qui va donner l'occasion au mouvement de s'épanouir, de se structurer et 
de s'exprimer politiquement. Tout semble alors possible, les initiatives fleurissent, tant militantes qu'institutionnelles $»^{7}$.

Les changements politiques ont des conséquences directes sur le monde de la CST. D'une part les lois Deferre participent à une décentralisation : «à l'époque, il s'agissait dans les esprits de rompre avec un centralisme qui éloignait les lieux de décision et de défendre les moyens de vivre et travailler au pays $»^{8}$. D'autre part, le ministre de la Recherche et de la Technologie Jean-Pierre Chevènement, favorise le développement d'un réseau régional ${ }^{9}$ de culture scientifique «qui préfigure l'actuel réseau des centres de culture scientifique et vient s'ajouter à la Cité des Sciences et de l'Industrie en construction $»^{10}$.

Les Assises de la Recherche et de la Technologie organisées à partir de 1981 par JeanPierre Chevènement permettent de formuler des projets - les CCST « offriront des espaces spécialisés (expositions, exploratoire, documentation, salles d'actualités, lieux de débats et de confrontations, planétarium...) afin de s'adapter à la diversité des motivations des citoyens [...] $»^{11}$. En 1982, la loi d'orientation et de programmation de la recherche, assigne notamment aux enseignants-chercheurs «la mise à disposition sociale de leurs travaux ». "C'est sur ces dispositions que vont s'appuyer un peu partout en France les militants pour développer leurs actions » soulignent Étienne Guyon et Bernard Maitte ${ }^{12}$.

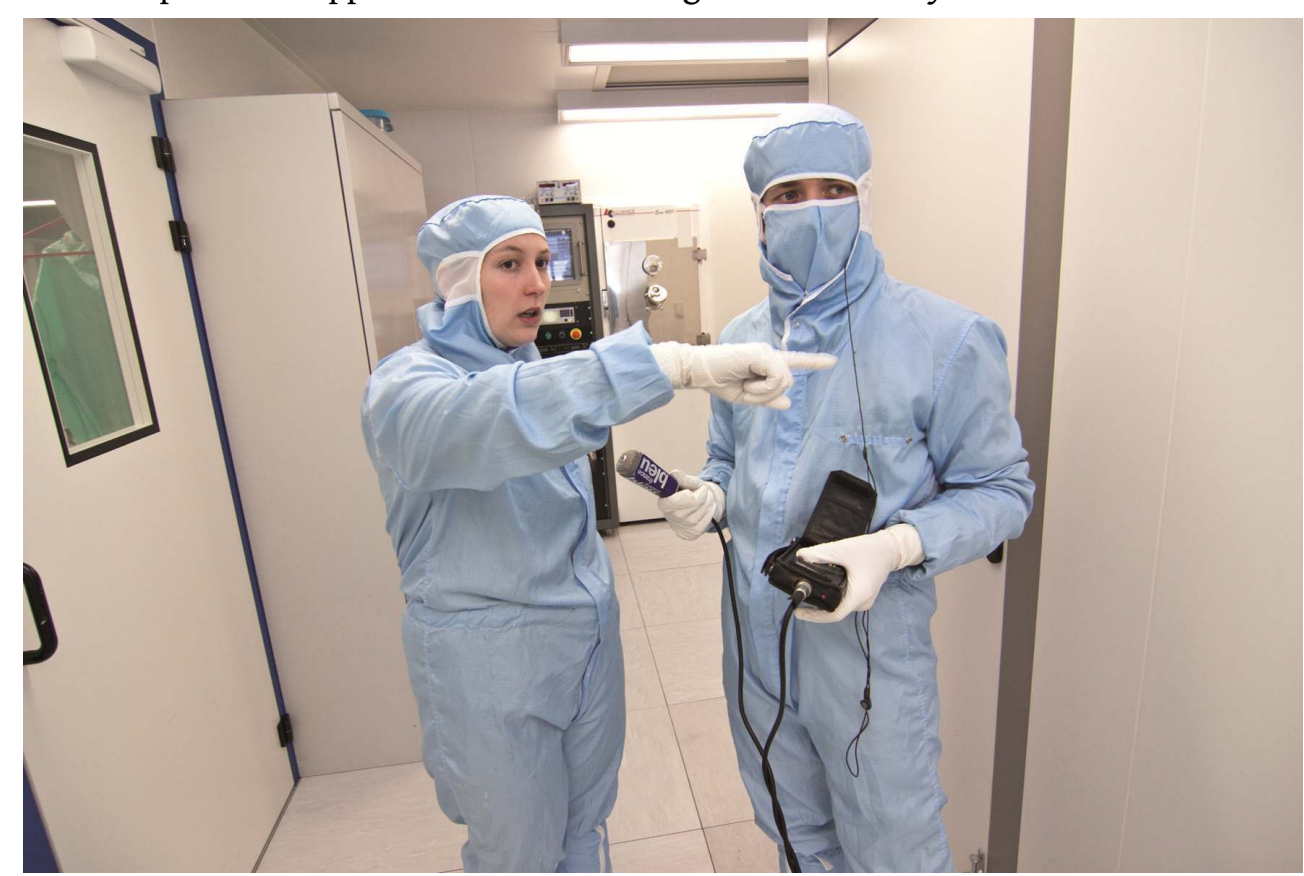

Depuis 2005, la Mission Culture Scientifique de l'université de Franche-Comté produit et réalise des interviews de chercheurs, qui sont diffusées le week-end sur France Bleu Besançon.

(C) UNIVERSITÉ DE FRANCHE-COMTÉ/LUDOVIC GODARD

Ce mouvement se développe sous l'influence de Hubert Curien (1924-2005), le ministre qui succède à Jean-Pierre Chevènement. Avec le soutien de ministères, de collectivités locales (départements, villes), parfois avec la mise en place des contrats de plan État/ Région, plusieurs Centres de Culture Scientifique, Technique et Industrielle (CCSTI) sont alors créés $^{13}$. Dès lors, le sigle CCSTI recouvre une hétérogénéité de configurations singulières en termes de tailles, de statuts et de sources de financements. Mais tous ces lieux, généralement dépourvus de collections, sont historiquement marqués par une volonté d'éducation. Lieux de médiation culturelle, ils se focalisent sur des 
muséographies didactiques et plusieurs de ces centres s'implantent là où sont leurs publics potentiels.

19 Alors que les collectivités locales s'engagent fortement aux côtés de ces structures, cellesci ont à cœur de montrer qu'elles peuvent toucher de nouveaux publics dans les territoires. Alex Querenet (1953-2008) évoque la création en 1987 de l'Association pour la Promotion de la Culture Scientifique, Technique et Industrielle Nord-Est Franche-Comté (qui deviendra Le Pavillon des sciences) en ces termes : « les élus affirment clairement la volonté de voir se développer, dans notre région, un programme qui vise à l'alphabétisation scientifique et technologique des habitants de l'Aire Urbaine. L'enjeu est de taille. En effet, si l'on considère la distance anormale entre le citoyen, l'homme de la rue et les aspects actuels du développement des sciences et techniques, il en résulte une urgente nécessité d'aider le public à se réapproprier cet élément essentiel de la culture moderne que doit être la familiarité avec les sciences et les techniques. De cet objectif dépend notre démocratie $»^{14}$. Ce projet ne vise pas uniquement à "populariser les sciences ", mais bien à contribuer à l'évolution du rapport sciences-citoyen dans un territoire donné.

Parallèlement, des associations d'éducation populaire développent des pratiques «hors les murs » orientées sur les sciences en utilisant des véhicules, pour aller à la rencontre des publics. Par exemple, l'association Planète sciences, créée en 1962 sous le nom Association nationale des clubs scientifiques, va à la rencontre des jeunes et leur propose des activités scientifiques notamment dans les domaines de l'espace et de la robotique.

Créée en 1992 par Hubert Curien La Science en Fête, qui deviendra La Fête de la Science, est un événement qui vise à promouvoir la science auprès du grand public. Elle trouve des déclinaisons sur l'ensemble du territoire, en permettant aux acteurs de la recherche, ainsi qu'aux associations d'amateurs, d'aller à la rencontre des publics.

\section{Outils nomades traditionnels}

Ces quarante dernières années, de nombreux outils, pour des pratiques « hors les murs » ont été développés pour irriguer les territoires.

Les premiers sont les expositions adaptées aux lieux culturels rencontrés sur les territoires. Ces lieux sont les salles d'expositions des CCSTI, des musées ou des centres culturels, mais aussi les salles de classe ou les médiathèques. Il existe les expositions " panneau » mais aussi « volume ». Par exemple, dans le Nord-Pas de Calais, l'association Alias (qui deviendra le Forum des sciences) réalise des modules d'expositions itinérants, « des valises-explorations, inspirées à la fois de celles de la Fondation 93, des expositions à tiroirs du Bauhaus et des manipulations des cabinets de curiosités, circulent entre différentes structures éducatives de la région et sont transportées dans des coffres de voitures $»^{15}$. À Orléans, Centre Sciences développe des expositions itinérantes légères à destination notamment d'autres pays francophones. À la fin des années 1990, la Cité des Sciences et de l'Industrie a aussi expérimenté les «Inventomobiles », qui sont des modules itinérants intégrant des expositions, avec des thèmes traités à la Cité des Enfants, et du matériel pédagogiques en lien avec les programmes scolaires. 


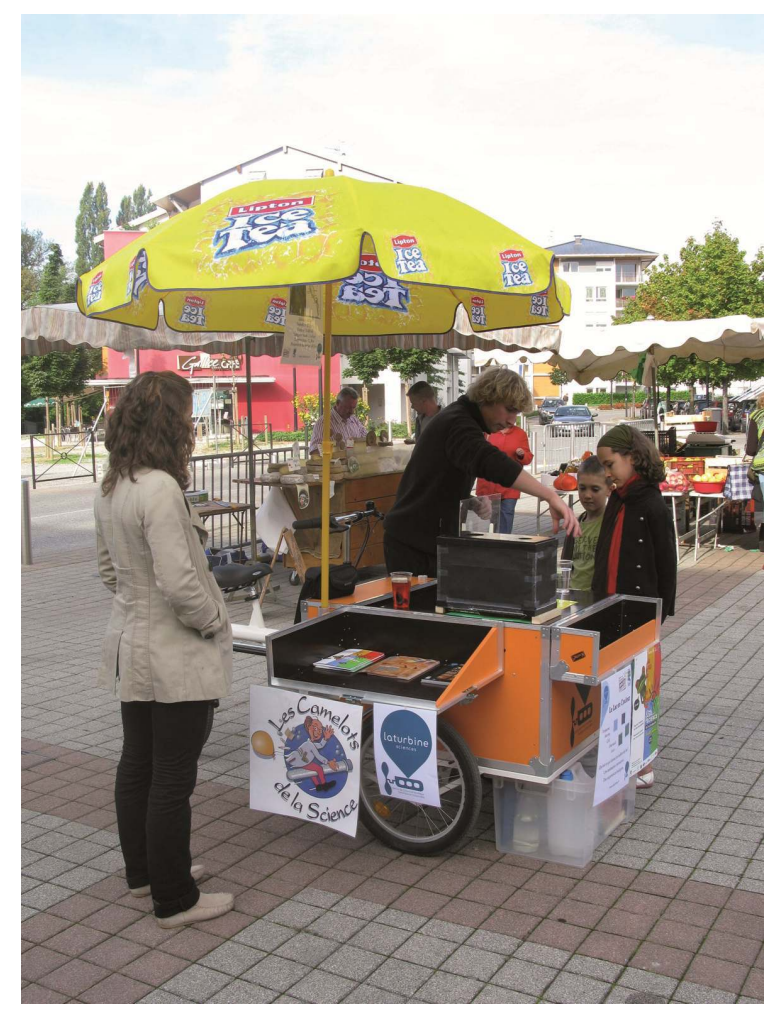

Le Triporteur de La Turbine et Les camelots de la science, sur le marché de Cran-Gevrier (C) LA TURBINE

Le développement de ces outils est étroitement lié aux canaux existant pour irriguer les territoires. Il s'agit de réseaux de communication (routes, canaux et voies ferrées) et de diffusion (écoles, collèges, lycées, médiathèques, comités d'entreprise, associations) existants et qui maillent finement les territoires.

Ces outils nomades peuvent associer un animateur, un véhicule et un objet de médiation. Le véhicule peut être un vélo. Dans ce cas, les médiateurs utilisent des triporteurs pour aller à la rencontre des publics et, sur un plateau, ils utilisent des objets qui permettent aux publics de réaliser les expériences par eux-mêmes.

Les véhicules utilisés pour des actions de médiation «hors les murs » peuvent être aussi des camionnettes, des camions, des bus, des trains, des péniches ou même des pirogues. Leur objectif est d'aller à la rencontre de publics sans cesse renouvelés, en investissant des lieux qui leurs sont familiers. La présence de ces supports, y compris du fait de leur détournement d'usage, contribue à créer l'événement.

À l'échelle de la Région et de l'inter Région, a été développé aussi le concept des Colporteurs des sciences. Création du CCSTI Franc-Comtois, le colporteur est un outil adapté à son territoire. En supprimant l'obstacle d'un déplacement de groupe, particulièrement pour les élèves de primaire et collèges, dont les coûts de transports sont de moins en moins pris en charge, il permet de remplir une mission régionale et d'intervenir plus particulièrement en milieu rural. L'idée, qui s'enracine dans une culture régionale, les montagnes jurassiennes ayant été parcourues par des "colporteurshorlogers ", est simple : il associe un animateur, un véhicule utilitaire et un objet mobile de découverte. Il repose sur deux principes de base : l'itinérance (pour aller là où sont les publics) et l'expérimentation (privilégier la démarche, la pédagogie active de type atelier). Ce concept a été repris dans le Massif du Jura à Saignelégier (Suisse), en Alsace, 
par La Nef des sciences et dans d'autres régions à dominante rurale auxquelles il est très adapté.

Si la fonction de ces véhicules est de transporter le matériel, ils peuvent également accueillir du public et se transformer alors en espace d'exposition ou d'animation, le détournement d'usage prend alors tout son sens. Ces outils de médiation « hors les murs » se révèlent être parallèlement d'excellents instruments de promotion pour les structures, en donnant envie aux visiteurs de venir ensuite dans ces lieux plus classiques.

\section{En 1986, à Pleumeur Bodou, l'Abret (devenu Armor}

Science) a mis sur les routes deux petites camionnettes pour transporter des expositions panneaux et un planétarium puis, deux ans plus tard, Roule la science, une remorque de 12 mètres de long. Actuellement, Sciences Animation, le CCSTI de Toulouse met en place le Média Mobile. Cet outil itinérant repose sur un semi-remorque constitué d'un plateau entièrement modulable. Il est équipé des technologies numériques et intègre tous les moyens de production et de diffusion participatifs pour permettre la réalisation de contenus avec le public. En Lorraine, il s'est avéré que la péniche était un outil adapté, pour parcourir la Région Lorraine, jusqu'à la frontière du Luxembourg. La péniche des sciences a été lancée en 1989-1990 à Thionville. Cette dernière a circulé dans le Nord-Est de la France, sur la Seine puis dans le Sud du territoire. Depuis 2005, les CCSTI de Laval et Sablé-sur-Sarthe proposent au public de découvrir Exp'Eau, une exposition sur le thème de «l'eau, milieu de vie », en montant à bord de la péniche Science Escale.

Ces outils permettent de balayer un territoire plus large, de transporter un matériel plus important que le colporteur et parfois d'accueillir du public.

\section{Investissements d'Avenir}

Depuis 2011, les projets soutenus par le Commissariat Général aux Investissements d'Avenir, pour le Développement de la culture scientifique et technique et l'égalité des chances, ont favorisé l'émergence de projets innovants, dont certains étroitement liés aux territoires.

Parmi les projets lauréats, plusieurs visent à mettre en place des actions sur l'ensemble du territoire national, certains à développer des actions interrégionales et d'autres à renforcer une structuration régionale ou même locale, à l'échelle d'une ville ou d'une agglomération, de l'offre en matière de culture scientifique. Certaines actions visent des publics spécifiques (scolaires, public jeune, enseignants), d'autres le grand public.

4 Parmi les projets à vocation régionale, le projet Construire Ensemble une Région de la Connaissance (Cerco) a pour ambition de construire, à l'échelle de la Lorraine, un maillage territorial fin en fédérant la plupart des acteurs rassemblés au sein du projet Hubert Curien. De même, Parcours en sciences et techniques pour la réussite des jeunes ligériens, a pour ambition de donner accès à la culture scientifique, technique et industrielle aux jeunes des Pays de la Loire, durant leur parcours de vie et de leur donner des perspectives professionnelles.

Le projet Innovation pour la Médiation dans les Territoires (Inmediats), dont l'un des objectifs est de « réduire les distances sociales, culturelles et géographiques en proposant des modes d'accès innovants à des contenus scientifiques", rassemble des acteurs répartis sur le territoire national. Il s'appuie notamment sur les technologies numériques 
et s'adresse aux jeunes de 15 à 25 ans. Territoires de la CSTI, projet également à vocation interrégionale, s'oriente davantage vers les publics des territoires à dominante rurale et des villes petites et moyennes.

\section{Médias et outils innovants}

Les médias nationaux (C'est pas sorcier, On n'est pas que des cobayes à la télévision, La tête au carré, à la radio, le supplément «sciences » des quotidiens Le Monde, Libération ou Le Figaro) et régionaux (Ouest France, en partenariat avec l'Espace des sciences) contribuent également à la diffusion de la CST dans les territoires. Depuis 2005, la Mission culture scientifique de l'université de Franche-Comté produit et réalise des interviews de chercheurs qui sont diffusés le week-end sur France-Bleu Besançon. Cette chronique permet de présenter l'actualité des laboratoires francs-comtois à un large public, implanté sur un territoire à dominante rurale. Cette initiative ne relève ni du journalisme indépendant, ni de la communication institutionnelle, mais bien du champ de la culture scientifique.

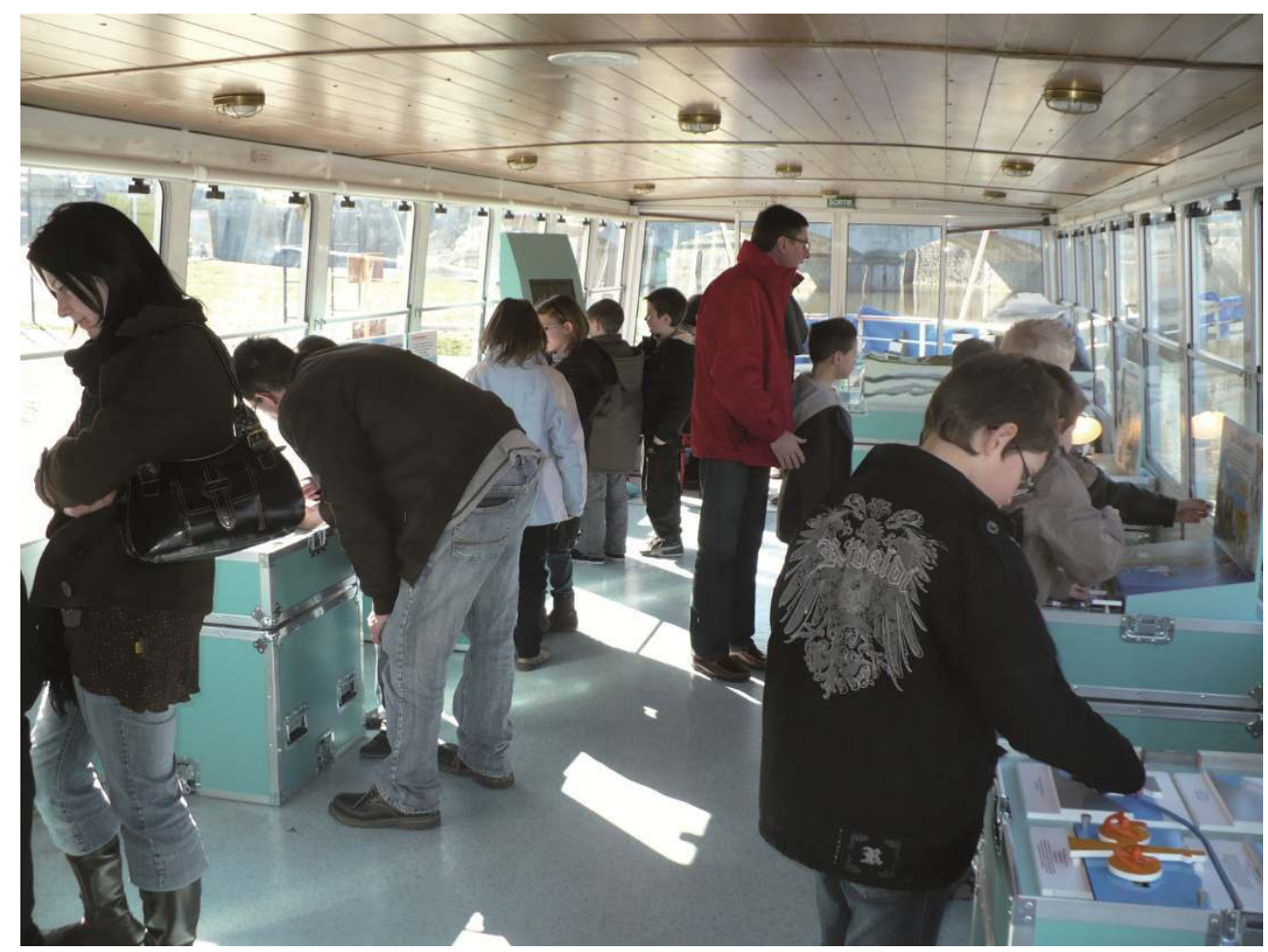

Les CCSTI de Laval et de Sablé-sur-Sarthe, se sont lancés dans l'aventure de Science Escale, une péniche scientifique régionale navigant sur 3 rivières (La Mayenne, la Sarthe et l'Oudon) : à bord, des expositions interactives et des ateliers sur le thème de l'eau.

(c) CCSTI de Laval 


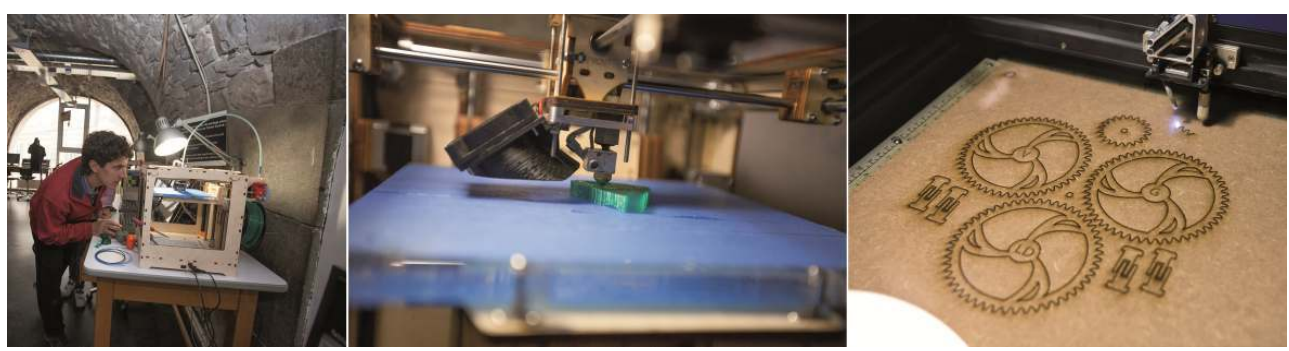

De nouvelles pratiques de médiation, qui associent les technologies numériques, mais aussi les modes de fonctionnement des communautés de l'innovation ouverte, sont développées dans les territoires. Un Fab User réalise des pièces et des rouages grâce à l'imprimante 3D et la découpeuse laser du Fab Lab de La Casemate à Grenoble

(c) CCSTI GRENOBLE-LA CASEMATE/PIERRE BORgHI

Les technologies numériques, mais aussi les modes de fonctionnement développés dans les réseaux sociaux et les communautés de logiciels libres et de l'innovation ouverte, permettent de proposer de nouvelles pratiques de médiation.

Parmi ces actions, dont plusieurs sont développées et évaluées dans le cadre du projet Inmediats, figurent notamment les FabLab, les Living Lab ou l'expérimentation et l'évaluation d'usages liés par exemple au développement d'univers virtuels immersifs ou de Serious games, s'appuyant sur des contenus scientifiques.

Le développement de ces outils part d'une réflexion sur les publics et leurs rapports aux sciences. Alors qu'une grande partie de la société se désintéresse des sciences, l'objectif est «de partir des centres d'intérêt des jeunes pour les conduire à s'intéresser à la pratique culturelle scientifique et technique $»^{16}$. Partant du constat que les nouvelles technologies engendrent de nouveaux moyens d'accéder à la connaissance, la stratégie est de «développer de nouveaux outils de médiation, de nouveaux services et de nouveaux contenus, offrant de nouvelles perspectives au champ de la culture scientifique ».

\section{Participatif}

Une initiative innovante revient sans doute aujourd'hui aux muséums. En effet, les programmes de sciences participatives, lient la production de connaissance, les publics et les territoires. Dans leurs domaines de prédilection - les sciences naturelles - plusieurs muséums proposent à leurs publics de contribuer à la recherche en découvrant, à l'aide de protocoles rigoureux, la biodiversité qui les entoure. L'originalité de la démarche - qui n'est pas sans rappeler celle des correspondants du Jardin du Roy du XVIII ${ }^{e}$ siècle - est de concerner autant des territoires urbains que la campagne.

41 Le rapport L'apport des sciences participatives dans la connaissance de la biodiversité, remis au ministre de l'Écologie en janvier 2012 par Gilles Bœuf, dresse un historique et un état des lieux précis des démarches entreprises pour mieux connaître la biodiversité et son évolution.

42 «Depuis ces dernières années, trois expressions dont le point commun est le mot science - science participative, science citoyenne, science collaborative - semblent vouloir se partager ce nouveau mode de participation du public et du grand public, avec souvent une absence de définition formelle, des frontières bien floues et, dans leur application, des nuances entre ces sciences pas toujours aisées à déceler. La caractéristique commune 
de ces trois expressions est de faire appel aux réseaux existants qui veulent bien s'impliquer et à toute personne volontaire qui accepte de suivre un protocole prédéterminé. Les données récoltées sont validées dans un premier temps par des têtes de réseau » souligne le rapport ${ }^{17}$.

Parmi les exemples de programmes avec données de suivi, figure Vigie-Nature, initié en 1989 par le département scientifique «Écologie et gestion de la biodiversité » du Muséum national d'Histoire naturelle. Ce dernier fédère des réseaux d'observateurs naturalistes volontaires. Il produit des indicateurs, des cartes, des bilans nationaux, qui constituent de véritables références scientifiques et permettent une meilleure compréhension de l'état de la biodiversité et de son fonctionnement.

Lancé en 1989 avec le Centre de recherches pour le baguage des populations d'oiseaux (CRBPO), le programme Suivi Temporel des Oiseaux Communs (STOC) fédère des ornithologues bénévoles et a pour objet de suivre l'évolution de populations d'oiseaux communs classées selon leur mode alimentaire. Des programmes concernant le dénombrement des papillons de jardin, les insectes pollinisateurs (suivi photographique des insectes pollinisateurs) et les plantes communes d'Europe (Vigie-Flore) ont également été lancés. Ce dernier programme a connu un large succès, puisqu'il a concerné 269 observateurs, dans 82 départements.

Les données de terrain locales, collectées par les observateurs volontaires sur l'ensemble du territoire sont ensuite reprises par les scientifiques. Elles participent à l'amélioration des connaissances sur la biodiversité ordinaire et s'insèrent ainsi dans des problématiques plus larges, liées par exemple au changement climatique ${ }^{18}$.

Même si le rapport de Gilles Bœuf soulève la question de la validation des données et de méthodologies expérimentales parfois difficiles à mettre en œuvre sur le terrain, il précise dans sa conclusion: «aujourd'hui, le développement des sciences participatives est un fait de société et le combat permanent pour sauver la biodiversité en est imprégné ».

\section{Publics}

Une meilleure connaissance des territoires passe inévitablement par la construction d'une cartographie détaillée des publics qui le composent.

Parmi les exemples d'études récentes, figure la recherche menée par Boris Urbas. Depuis 2009, ce dernier développe une thèse en Sciences de l'information et de la communication, au sein du laboratoire Communications, médiations, organisations, savoirs (Cimeos) de l'université de Bourgogne, autour des publics du CC-STI de FrancheComté.

49 Pourquoi franchir le seuil d'un tel établissement alors que l'accès à des contenus scientifiques est aujourd'hui possible par des médias spécialisés, par le Web? Pour répondre à des questions de ce type, le doctorant analyse la place des savoirs scientifiques par rapport aux pratiques culturelles, en s'intéressant plus particulièrement aux visiteurs individuels et à la situation de médiation à proprement parler. L'objectif est d'améliorer la connaissance des publics, de la relation à la structure à ses publics et publics potentiels, et contribuer ainsi à une réflexion sur la notion de publics et de «non-publics » dans le domaine de la communication des sciences et techniques. "Cette sensibilisation de la population à la culture scientifique recouvre des pratiques communicationnelles 
différenciées, renvoyant à des collaborations dans une inscription territoriale et générant leurs propres publics; elles induisent la production de situations de communications spécifiques (des formats de médiation) » ${ }^{19}$.

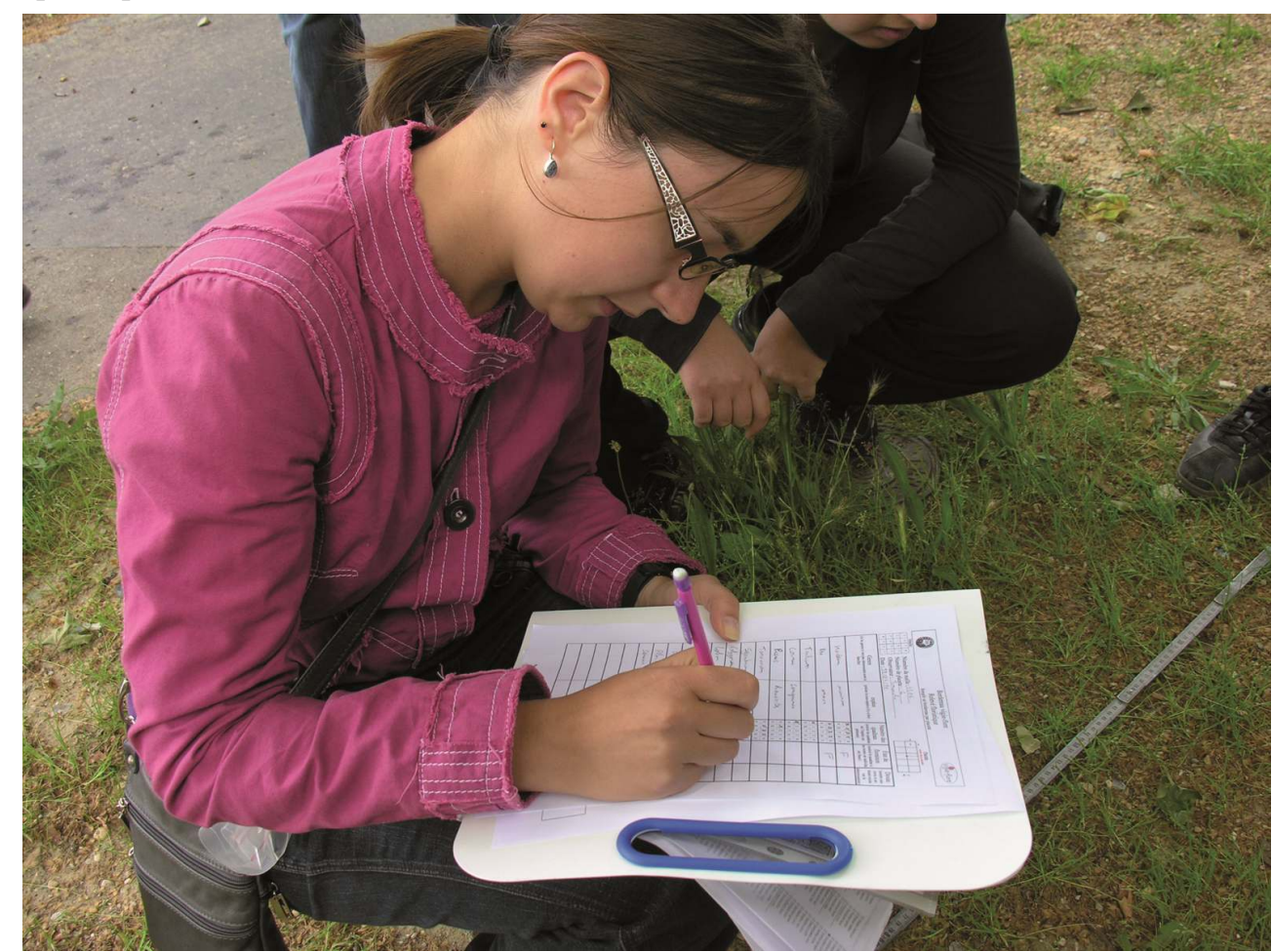

Relevé floristique réalisé dans le cadre de Vigie-Flore, lancé en 2009 par le laboratoire de Conservation des espèces, restauration et suivi des populations (CERSP) du Muséum national d'Histoire naturelle et qui propose aux botanistes amateurs de suivre l'évolution de l'abondance des espèces végétales les plus communes en France.

(C) R. NATTIER-TIMARCHA

Autre exemple récent, depuis 2009 l'office de Coopération et d'Informations Muséales (OCIM) a mis en place un Observatoire du Patrimoine et de la Culture scientifiques et techniques (OPCST) avec l'objectif de produire des synthèses chiffrées sur les institutions, leur organisation, leurs activités, leurs publics, et leurs collections sur le territoire. Ainsi, en s'appuyant sur les besoins formulés par les professionnels, les acteurs, les institutions, afin de mieux se situer dans l'exercice de leurs missions, l'OPCST a édité une série de synthèses. Celles-ci proposent des photographies du paysage national des activités de grandes familles institutionnelles (muséums, jardins botaniques, universités, CCSTI et assimilés) œuvrant dans le domaine de la culture scientifique et technique. À partir de ces synthèses, seront développées des analyses transversales et explorations territoriales permettant d'appréhender les dynamiques locales entre ces différents acteurs.

\section{Conclusion}

51 La question des territoires se place au cœur de la réflexion sur la mise en place actuelle de nouveaux schémas de gouvernance nationale et régionale. Les remaniements peuvent-ils contribuer à rapprocher les publics des centres de production du savoir, des lieux de médiation? 
52 L'histoire montre qu'il est nécessaire de travailler sur la pérennité des pratiques et les savoir-faire développés depuis trente ans dans les territoires par les professionnels de la culture scientifique technique et industrielle. Les savoir-faire acquis par les acteurs de terrain pour travailler avec des publics dit « éloignés » se révèlent être des outils efficaces contre les disparités sociales et territoriales. Cependant, les actions de médiation présentielles, souvent inscrites dans le temps de l'oralité de la rencontre avec le public, tout comme certaines pratiques de médiation « hors les murs », reposent sur un équilibre fragile. Les comprendre, les analyser, constitue la première étape de leur pérennité.

Alliées au développement de nouveaux outils, faisant notamment appel aux technologies numériques, ces pratiques permettent de faire évoluer la place du savoir scientifique dans la relation entre publics et territoires. Les programmes de sciences participatives, qui allient des pratiques héritées des naturalistes du XVIII ${ }^{\mathrm{e}}$ siècle et des outils contemporains, permettent à la fois un renforcement du lien entre la recherche et les populations, un maillage fin du territoire ainsi qu'une production de connaissances de référence sur l'évolution de l'état de la biodiversité. L'ouverture des sciences participatives aux sciences sociales sera sans doute une nouvelle étape dans l'activation de ce lien.

Il paraît important d'inventer de nouvelles formes de mutualisation entre les acteurs, ruraux ou urbains et entre les réseaux, associatifs, publics ou universitaires. L'objectif est de construire des réponses adaptées aux publics visés - c'est l'enjeu de la mise en place d'ateliers scientifiques depuis la rentrée 2013 dans le cadre de la réforme des rythmes scolaires $^{20}-$, et contribuer ainsi à l'évolution du rapport des publics aux sciences dans les territoires.

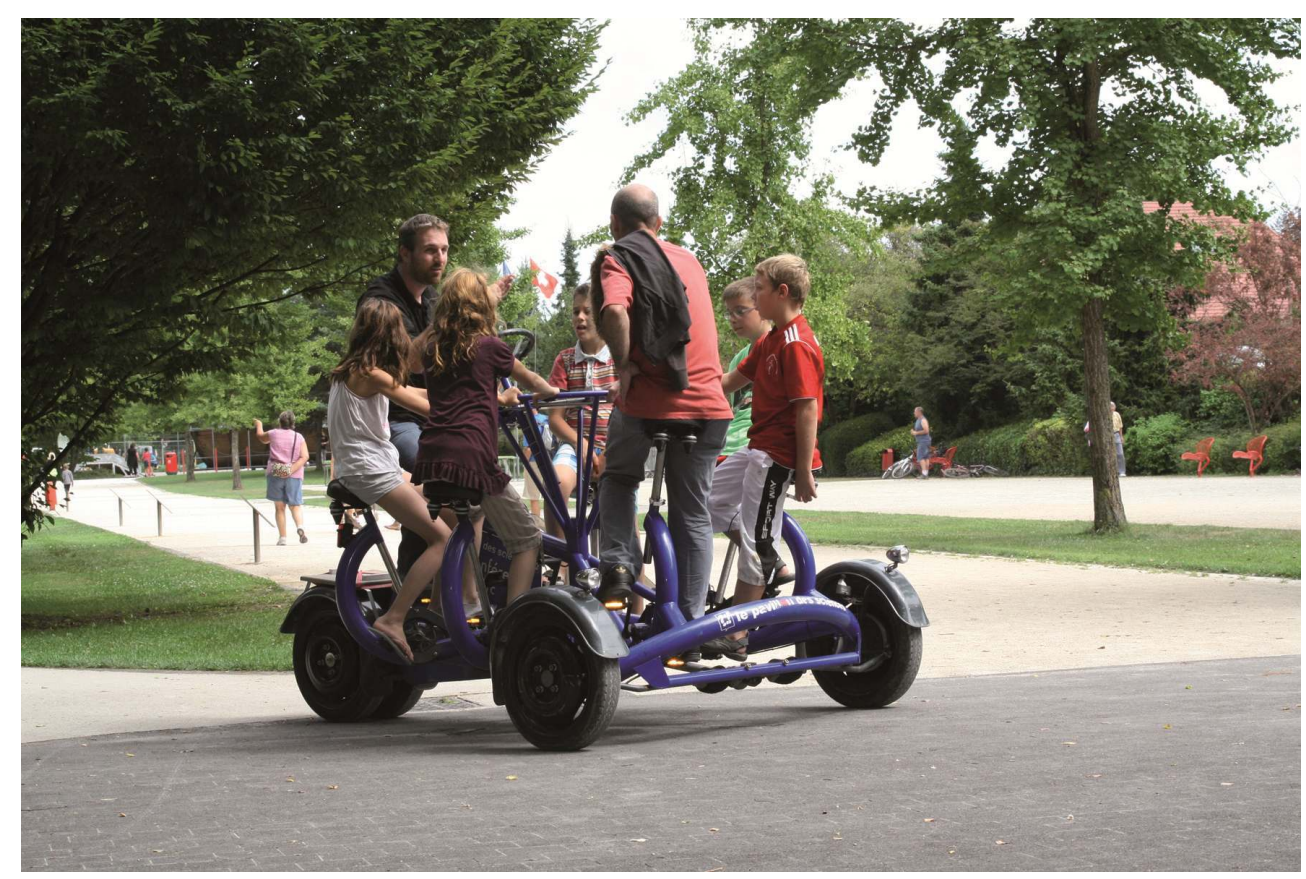

À Montbéliard, avec le Véloconférence, l'animateur scientifique et six passagers partent à la découverte de la faune, la flore mais aussi d'un environnement scientifique dans le parc du Près la Rose.

(C) Pavillon des sciences/P. Viennet

Répondre à cette ambition implique la création d'outils adaptés, mais aussi, et toujours dans une logique de complémentarité, l'activation de nouveaux réseaux. C'est pourquoi, dans le contexte de décentralisation, il est aussi indispensable de créer des liens avec les 
acteurs d'autres politiques publiques du territoire, liés par exemple à la santé ou à l'alimentation.

Si la culture scientifique, technique et industrielle se donne pour projet de contribuer à l'émergence de citoyens émancipés et épanouis plus nombreux et de prendre part au développement de la société de la connaissance, il est aussi nécessaire que les besoins de ces citoyens soient aussi entendus et écoutés. Cette ambition passe par la prise en compte des spécificités de leurs territoires.

\section{NOTES}

1. Projet de loi relatif à l'enseignement supérieur et à la recherche. Assemblée Nationale, session extraordinaire, 9 juillet 2013.

2. Levy-Leblond, J.-M. La Culture scientifique et technique : entre le mot et la chose, entretien réalisé par M. Barrère, La Recherche, $\mathrm{n}^{\circ}$ 156, 1984.

3. La muséologie selon Georges-Henri Rivière. Éditions Dunod, 1989, 402 p.

4. Guyon, É. et Maitte, B. Le Partage des savoirs scientifiques, Les Centres de culture scientifique, technique et industrielle, La Revue pour l'histoire du CNRS, $n^{\circ} 22,2008$.

5. Guyon, É. et Maitte, B. Op. cit.

6. Bergeron, A. Patrimoine et culture scientifique: sur l'inscription culturelle des savoirs. Patrimoine et communautés savantes. Presses Universitaires de Rennes, 2009, pp. 209-223.

7. Guyon, É. et Maitte, B. Op. cit.

8. Bergeron, A. Op. cit.

9. Andrée Bergeron cite le «Discours de clôture au colloque national sur la Recherche et la Technologie » de Jean-Pierre Chevènement, janvier 1982, Actes du Colloque national Recherche et Technologie. Paris : Éditions du Seuil, 1982, p. 204.

10. Bergeron, A. Op. cit.

11. Guyon, É. et Maitte, B. Op. cit.

12. Guyon, É. et Maitte, B. Op. cit.

13. En 1983, le IX ${ }^{\mathrm{e}}$ plan, dans son programme prioritaire d'exécution $\mathrm{n}^{\circ} 3$, indique dans le paragraphe Promouvoir la culture et l'information scientifique et technique: «Un réseau de centre de culture scientifique et technique sera mis en place dans les régions, articulé avec le musée de La Villette ». C'est alors que la Midist (mission interministérielle) ajoute le « $\mathrm{i}$ » de « industriel » aux CCST (Guyon et Maitte, 2008).

14. Querenet, A. Vers une Europe de la Culture scientifique Nord-Est-Franche-Comté, Bulletin de l'Association des écrivains scientifiques de France, n 41 , octobre 1992, pp. 35-41.

15. Guyon, É. et Maitte, B. Op. cit.

16. Amourous, T. et Boilève, M. Inmediats, Acte 1. 2013, 56 p.

17. Bœuf, G., Allain, Y.-M. et Bouvier, M. L'apport des sciences participatives dans la connaissance de la biodiversité. Rapport remis à la ministre de l'Écologie en janvier 2012, 27 p.

18. Sur ce sujet voir "Citoyenneté et biodiversité : une réflexion sur les sciences participatives » le numéro thématique ( $\left.\mathrm{n}^{\circ} 144\right)$ de La Lettre de l'OcIM publié en décembre 2012 en collaboration avec le Muséum national d'Histoire naturelle et Espace pour la vie Montréal.

19. Urbas, B. De la recherche du «non-public» d'un CCSTI à l'étude des appropriations informelles des sciences et techniques: quelles méthodologies? Actes des journées doctorales 
2011. Bordeaux, 30-31 mars 2011. Société française des sciences de l'information et de la communication, pp. 225-232.

20. Décret $n^{\circ}$ 2013-77 du 24 janvier 2013, relatif à l'organisation du temps scolaire dans les écoles maternelles et élémentaires.

\section{RÉSUMÉS}

Comment réduire la distance, autant géographique que sociale, entre les publics, les lieux de productions du savoir et les lieux de médiation? Dans un paysage de la culture scientifique, technique et industrielle en mutation - induite autant par la mise en œuvre de l'acte III de la décentralisation, l'émergence de projets soutenus par le Commissariat Général à l'Investissement ou le développement de pratiques ou d'outils de médiation inédits - un nouveau rapport au territoire se dessine.

\section{INDEX}

Mots-clés : Territoires, culture scientifique

\section{AUTEUR}

\section{SAMUEL CORDIER}

Samuel Cordier est directeur du Pavillon des sciences, CCSTI de Franche-Comté.

samuel@pavillon-sciences.com 